\title{
Cool to Critical: Managing Web Services Now
}

\author{
Ellen Stokes \\ Senior Technical Staff Member \\ IBM/Tivoli Systems Management \\ 9442 Capitol of Texas Highway North, Austin, TX 78759, USA \\ stokese@us.ibm.com
}

Web services are no longer just hype - they are being sanctioned by the industry on two fronts, standards and products. IBM is investing time, talent, and money on both these fronts, establishing itself as an industry leader. Web services are being developed as the foundation of a new generation of business-to-business and application integration architectures. This places Web services technologies in a business critical role within most enterprises. The corollary to this is that the Web services and the applications that use them must be manageable, from end to end, through the firewalls. The business-to-business Web services applications require management solutions in kind. They must be platform and technology agnostic, available through firewall, internet friendly, and flexible. This presents new challenges and opportunities to management vendors.

This session will explain IBM's view of the challenges and vision for managing Web services as well as leveraging Web services for management purposes. The session will also talk about how the same management challenges and solutions might be applied to Grid Services. In summary, this session will address IBM's current work in the Web Services management area and how IBM's products address the needs of e-business and management through Web Services. 